BMJ

Open

Gastroenterology

\title{
Risk of common infections in people with inflammatory bowel disease in primary care: a population-based cohort study
}

\author{
Peter M Irving (D) , ${ }^{1,2}$ Simon de Lusignan, ${ }^{3,4}$ Daniel Tang, ${ }^{5}$ Monica Nijher, ${ }^{5}$ \\ Kevin Barrett ${ }^{6}$
}

\begin{abstract}
To cite: Irving PM, de Lusignan S, Tang D, et al. Risk of common infections in people with inflammatory bowel disease in primary care: a population-based cohort study. BMJ Open Gastro 2021;8:e000573. doi:10.1136/ bmjgast-2020-000573
\end{abstract}

- Additional material is published online only. To view please visit the journal online (http://dx.doi.org/10.1136/ bmjgast-2020-000573).

Received 12 November 2020 Revised 20 January 2021 Accepted 31 January 2021

Check for updates

(C) Author(s) (or their employer(s)) 2021. Re-use permitted under CC BY-NC. No commercial re-use. See rights and permissions. Published by BMJ.

${ }^{1}$ Department of Gastroenterology, Guy's \& St Thomas' Hospital, London, UK ${ }^{2}$ School of Immunology and Microbial Sciences, King's College London, London, UK ${ }^{3}$ Nuffield Department of Primary Care Health Sciences, University of Oxford, Oxford, UK

${ }^{4}$ Royal College of General

Practitioners (RCGP) Research and Surveillance Centre (RSC), London, UK

${ }^{5}$ Pfizer Ltd, Tadworth, UK ${ }^{6}$ New Road Surgery, Croxley Green, UK

Correspondence to Dr Peter M Irving; peter.irving@gstt.nhs.uk

\section{ABSTRACT}

Objective To evaluate the risk of common infections in individuals with inflammatory bowel disease (IBD) [ulcerative colitis and Crohn's disease] compared with matched controls in a contemporary UK primary care population.

Design Matched cohort analysis (2014-2019) using the Royal College of General Practitioners Research and Surveillance Centre primary care database. Risk of common infections, viral infections and gastrointestinal infections (including a subset of culture-confirmed infections), and predictors of common infections, were evaluated using multivariable Cox proportional hazards models.

Results 18829 people with IBD were matched to 73 316 controls. People with IBD were more likely to present to primary care with a common infection over the study period ( $46 \%$ vs $37 \%$ of controls). Risks of common infections, viral infections and gastrointestinal infections (including stool culture-confirmed infections) were increased for people with ulcerative colitis and Crohn's disease compared with matched controls (HR range 1.121.83, all $p<0.001)$. Treatment with oral glucocorticoid therapy, immunotherapies and biologic therapy, but not with aminosalicylates, was associated with increased infection risk in people with IBD. Despite mild lymphopenia and neutropenia being more common in people with IBD (18.4\% and $1.9 \%$, respectively) than in controls $(6.5 \%$ and $1.5 \%$, respectively), these factors were not associated with significantly increased infection risk in people with IBD. Conclusion People with IBD are more likely to present with a wide range of common infections. Health professionals and people with IBD should remain vigilant for infections, particularly when using systemic corticosteroids, immunotherapies or biologic agents. Trial registration number Clinicaltrials.gov (NCT03836612).

\section{INTRODUCTION}

Ulcerative colitis (UC) and Crohn's disease (CD) are chronic inflammatory conditions collectively termed inflammatory bowel disease (IBD). ${ }^{1}$ Both conditions can follow a relapsing-remitting course or be continuously active. The disease spectrum ranges

\section{Summary box}

What is already known about this subject?

- Although people with inflammatory bowel disease (IBD) are known to be at increased risk of infection, the burden of common infections among people with IBD in primary care has not been clearly established.

What are the new findings?

- Using the UK primary care infectious disease sentinel network, we demonstrate people with IBD are at increased risk of common infections including gastrointestinal and viral infections, and that risk is highest in those managed with systemic corticosteroids, immunotherapies and biologic therapy.

How might it impact on clinical practice in the foreseeable future?

- Clinicians should be aware of the higher risk of common infections in people with IBD in primary care, particularly in the context of the ongoing COVID-19 pandemic.

from a quiescent state with few or no symptoms to potentially fatal disease. Medical intervention is often required over decades of disease and may include surgery. ${ }^{2}$ With the global burden of IBD rising, calls for improved treatment options ${ }^{4}$ have resulted in the development of several immunomodulatory and biologic therapies. ${ }^{1}$

Current treatment strategies for many people with IBD focus on immunomodulation. ${ }^{5}$ Though frequently effective for disease control, such treatments increase infection risk, ${ }^{67}$ including common and atypical pathogens. ${ }^{7}$ Understanding the infection risk in a community-based population with IBD has been brought into sharp focus recently with the onset of the COVID-19 pandemic. The need to protect vulnerable individuals led to recommendations from the British Society of Gastroenterology for people with IBD that was, in part, driven by medication use. ${ }^{8}$ 
However, decisions around which individuals required shielding were generally opinion based, largely due to the lack of data around the risks of common infections in people with IBD and their relationship with common comorbidities and medication use. It is notable that coding of comorbidity, the biggest risk factor for COVID19-related mortality, is more complete in primary than secondary care, ${ }^{9}$ and that medications and common infections are not systematically coded in secondary care, thus reinforcing the importance of studying infection risk in the primary care setting. Despite this, existing literature on infection rates in IBD has focused predominantly on secondary care populations. ${ }^{10-12}$ Although an increased risk of specific infections including herpes zoster and pneumonia has been observed in primary care, ${ }^{13} 14$ the total burden of common infection in individuals with IBD in primary care remains unknown. Similarly, while lymphopenia and neutropenia are known markers of infection risk in the general population, ${ }^{15}$ their utility as risk factors for common infections in people with IBD in the primary care setting has not previously been evaluated.

This study aimed to describe the risk of common infections, in a primary care population of individuals with UC and $\mathrm{CD}$, compared with a matched control population. Risk factors for common infections in IBD, with a focus on comorbidity, medication, lymphopenia and neutropenia, were explored.

\section{MATERIALS AND METHODS \\ Study design}

We performed a retrospective, matched cohort study to evaluate infection risk among people with IBD compared with population controls in UK primary care. The study protocol has been previously published. ${ }^{16}$

\section{Data source}

The Oxford-Royal College of General Practitioners (RCGP) Research and Surveillance Centre (RSC) network database was used. This UK-based database comprises pseudonymised primary care records of individuals registered with a large network of general practices, providing a representative sample of the primary care population in England. ${ }^{17}$ At the time of data extraction, RCGP RSC contained data from 2 million people, providing information on clinical diagnoses, anthropometric measurements, laboratory tests and prescriptions, coded using the Read coding system. ${ }^{18}$ UK primary care has been computerised since 1990s, and pay-for-performance data available from 2004 have resulted in high-quality clinical data entry about chronic disease. ${ }^{19} 20$ Studies using RCGP RSC have been published across many chronic diseases. ${ }^{21-24}$ RCGP RSC is the primary infectious disease sentinel network for the UK, providing weekly infections data since 1967 to monitor trends in infectious disease and investigate real-world vaccine efficacy. ${ }^{25}{ }^{26}$ General practices within the network receive feedback on their coding of infectious diseases, which designates cases as first, new or ongoing, thereby differentiating incidence from prevalence. ${ }^{27}$

\section{Study population}

People aged $\geq 18$, registered with a GP practice contributing to the RCGP RSC between 1 January 2014 and 1 January 2019, were eligible for inclusion.

\section{Definition of the exposed cohort with IBD}

People with an existing or incident diagnosis of UC or $\mathrm{CD}$, as defined by the presence of at least one diseasespecific Read code, were eligible for inclusion in the IBD cohort. Use of diagnosis codes alone has been reported to have a $97 \%$ positive predictive value for identifying IBD from electronic health records in the USA ${ }^{28}$ and has been validated in UK primary care as a means of identifying IBD. ${ }^{29}{ }^{30}$ Read codes used to identify UC and CD were based on the codes used by Abrahami et al, ${ }^{30}$ mapped to both Read code versions used within the RCGP RSC (online supplemental appendix 1). Start of follow-up for an individual with IBD was defined as the latest of 1 January 2014 or the date of IBD diagnosis.

\section{Definition of the matched unexposed cohort without IBD}

Individuals with a diagnosis of IBD were matched (nearest neighbour matching, with replacement) at their index date (start of follow-up date) with four unexposed individuals at general practice level by current age (per year), sex and time since practice registration. Eligible unexposed individuals at each index date comprised people actively registered at that date with no IBD history and a minimum 1 year registration with their RCGP RSC practice (to minimise the risk they had a non-recorded existing IBD diagnosis). Follow-up for each matched individual was started on the index date of their matched case with IBD. People diagnosed with IBD after the study start date were included in the pool of eligible unexposed individuals, but if matched, they were censored on the date of their IBD diagnosis. This meant individuals were eligible to contribute to unexposed person time prior to an IBD diagnosis. The end of follow-up was defined as the earliest of the study end date (1 January 2019), the date of patient transfer from an included general practice, date of death or the date an individual first developed an infection of interest. Follow-up for the unexposed cohort also ended if they developed IBD, at which point they became eligible for the exposed group. Individuals contributing at least 1 day of follow-up time were included in this study.

\section{Outcomes}

\section{Infections}

Infection outcomes were defined as the incidence of a new presentation of (1) common infection, (2) viral infection and (3) gastrointestinal (GI) infection during the study period. First or new presentations of an infection are coded accordingly in the database, enabling differentiation of new infections from chronic infections or 
follow-ups for the same episode. Common infection was defined as a composite of upper respiratory tract infections (URTI), pneumonia, acute bronchitis, influenzalike illness (ILI), skin infections, herpes simplex and herpes zoster infections, genital infections, urinary tract infections (UTI) and GI infections. Read codes used to identify common infections were taken from validated indicators used in routine surveillance by the RCGP RSC. Viral infections were defined as a composite of ILI, herpes simplex and herpes zoster infections and any upper or lower respiratory tract infections specifically coded as being viral. GI infection comprised clinical diagnoses of gastroenteritis, enteritis or infective colitis as well as laboratory-confirmed viral, bacterial or protozoan GI tract infections. The incidence of all infection subtypes comprising the primary outcome of common infection was examined in a secondary analysis. To distinguish GI infection from non-infective diarrhoea within the cohorts, a subanalysis of stool culture-confirmed GI infections (a composite of Clostridium difficile, Salmonella, Shigella and Campylobacter infections [online supplemental appendix 2]) was performed.

\section{Baseline measures}

Baseline measures comprised sociodemographic characteristics and clinical features and biomarkers associated with IBD or infection risk. Socioeconomic status was defined using the official national measure, the index of multiple deprivation ${ }^{31}$ and calculated using patient postcode, with the resultant scores stratified by deprivation quintile. Ethnicity was grouped into white, black, Asian, mixed and others. ${ }^{32}$ Body mass index (BMI), smoking status and alcohol use were defined using the most recent recorded data prior to the study start date. For these measures, we used the missing indicator variable method; the robustness of this approach was tested using sensitivity analysis (see below). Diagnostic codes were used to define the following baseline comorbidities (with the absence of a code for a comorbidity assumed to represent the absence of that comorbidity): diabetes mellitus, hypertension, hyperlipidaemia, atrial fibrillation, angina, myocardial infarction, congestive heart failure, peripheral vascular disease, stroke, transient ischaemic attack, chronic kidney disease stages 3-5 (CKD), dementia, rheumatoid arthritis, chronic liver disease, asthma, chronic obstructive pulmonary disease (COPD), joint replacement and fractures. Lymphocyte and neutrophil counts were defined as continuous measures, and with lymphopenia and neutropenia, subgroups were defined, respectively, by cut-offs of absolute lymphocyte count $<1.0 \times 10^{9}$ cells $/ \mathrm{L}$ and absolute neutrophil count $<1.5 \times 10^{9}$ cells $/$ L. $^{33} 34$ The following medications used in the management of IBD were examined: rectal 5-aminosalicylic acid (5-ASA), rectal glucocorticoids, oral 5-ASA, oral glucocorticoids, nonbiologic immunosuppressants and biologic therapies (online supplemental appendix 3). Baseline medication use was defined as the issue of a prescription 3 months before, to 30 days after, the study start date; information on whether prescriptions were dispensed is not captured in the RCGP database.

\section{Statistical analyses}

\section{Risk of infection in IBD}

Incident cases comprised individuals with a first-ever diagnostic Read code for infection during the study period. Incidence of common, GI and viral infections were calculated separately for UC and $\mathrm{CD}$, by dividing the number of incident cases by the sum of person-years of follow-up for the total eligible population over the study period and expressed as the number per 100 person-years. Risk of infection in UC and CD was estimated using unadjusted Cox proportional hazards models, stratified by matched set, to provide overall hazard ratios for each infection outcome. Models were subsequently adjusted for baseline measures using multivariable analysis.

\section{Predictors of infection in IBD}

Baseline factors associated with an increased risk of infection were evaluated in UC and CD separately using Cox proportional hazards models. Factors evaluated were age, sex, ethnicity, socioeconomic status, BMI, smoking, alcohol use, comorbidities, duration of disease and baseline medication use. To further evaluate the utility of lymphopenia and neutropenia as markers of infection risk, their time-varying association with risk of common infection over study follow-up was explored using unadjusted and adjusted Cox proportional hazards models, with each measure modelled as a continuous time-varying covariate. This time-varying approach maximises power and predictive ability compared with using baseline only measures. ${ }^{35}$ Test results recorded in the 2 weeks prior to an infection were excluded to reduce the likelihood the infection itself influenced the test result. As a sensitivity analysis, this exclusion window was extended to the 2 months prior to an infection. Time-updated lymphopenia status and neutrophilia status as binary covariates were examined using the same approach.

\section{Sensitivity analysis}

We ran the following post hoc sensitivity analysis to assess the robustness of finding for the primary outcomes: (1) repeating the primary analysis excluding matched controls without a least one recorded consultation in the year before cohort entry (to exclude practice nonattendees), (2) excluding patients with IBD registered within 1 year of the start of study follow-up (the same criteria as applied to controls in the primary analysis, to remove potentially more transient patents from the IBD cohort), (3) without adjustment for baseline medications that may be on the causal pathway between IBD and infection, (4) using a comorbidity count score in multivariable models as opposed to including each comorbidity as an individual term, (5) excluding IBD cases (and their matched controls) with missing data for ethnicity, BMI, smoking, alcohol use and deprivation. Statistical analysis was performed in R V.3.4.1. 


\section{RESULTS}

A total of 18829 people with IBD (UC n=11 360 and $\mathrm{CD} \mathrm{n}=7469$ ) (exposed cohort) were matched to 73316 people without IBD (unexposed matched cohort). 3118 $(16.6 \%)$ IBD cases were incident over the study period and $15711(83.4 \%)$ were diagnosed prior to the study start date. Age, sex, and follow-up time were closely matched (table 1). The cohort with IBD had a greater prevalence of several comorbidities including depression, rheumatoid arthritis, COPD, chronic liver disease and CKD (table 1). People with CD were younger, more likely to be underweight and had fewer comorbidities than those with UC.

\section{Primary analysis: people with IBD have an increased risk of common infection}

Of 8581 (46\%) people with IBD presented with common infection over the study period, compared with 27407 $(37 \%)$ in the control group. Event rates were higher in IBD being 16.4 (95\% CI 16.0 to 16.9 ) per 100 personyears in UC (table 2) and 17.4 (95\% CI 16.9 to 18.0) in CD (table 2), compared with 13.0 (95\% CI 12.8 to 13.2) per 100 person-years in the UC-matched cohort (table 2) and 12.7 (95\% CI 12.4 to 12.9) per 100 personyears in the CD-matched cohort (table 2). Event rates were similarly increased in IBD for viral infections and GI infections (table 2). Figure 1A and table 2 show an association between IBD and the three infection outcomes in unadjusted and multivariable-adjusted analyses. The association of greatest magnitude was for GI infections (adjusted Hazard ratio (aHR) 1.46 (95\% CI 1.26 to 1.69) for UC and aHR 1.83 (95\% CI 1.56 to 2.15) for $\mathrm{CD}$ ).

\section{Secondary analysis: risk for infection subtypes}

Figure $1 \mathrm{~B}$ shows the association between $\mathrm{UC}$ and $\mathrm{CD}$ and time to presentation of each infection subtype. Event rates and aHRs for each infection subtype are reported in online supplemental table 1. URTI, acute bronchitis and skin infections were the most common infections. Associations of the greatest magnitude were stool culture-confirmed Clostridium difficile, Salmonella, Shigella and Campylobacter infections and herpes zoster for UC, whereas for $\mathrm{CD}$, pneumonia and herpes zoster were most common.

\section{Secondary analysis: predictors of common infection}

Sociodemographic factors and pre-existing comorbidities

In $\mathrm{UC}$ and $\mathrm{CD}$, a higher risk of infection was seen in women, people of Asian ethnicity, the overweight or obese and current and ex-smokers (online supplemental table 2). In $\mathrm{UC}$ and $\mathrm{CD}$, the presence of depression, dementia, malignancy, COPD and myocardial infarction were associated with an increased risk of common infection. In individuals with UC, type 2 diabetes, CKD, stroke, angina and atrial fibrillation were additional significant risk factors.

\section{Medication exposure}

Oral glucocorticoid therapy, immunotherapies and biologic use were all associated with an increased risk of common infection to a similar degree in $\mathrm{UC}$ and $\mathrm{CD}$ (figure 2). As expected, rectal glucocorticoid use was higher in UC than CD (table 1), but the increase in risk of common infection with these medications was observed only in UC (figure 2).

\section{Lymphocyte and neutrophil count}

Of $15290(81 \%)$ people with IBD had a minimum of one lymphocyte count and neutrophil count during the study period (mean number of counts: 6 per person), compared with $44324(59 \%)$ people in the control cohorts (mean counts: 4 per person). Figure 3 shows that, in continuous analysis, a lower lymphocyte count was associated with an increased risk of common infection in people without IBD but not in those with IBD. When analysed by lymphocyte count status (where lymphopenia was defined as a lymphocyte count $<1.0 \times 10^{9}$ cells $/ \mathrm{L}$ ), $18.4 \%$ of the IBD cohort had at least one episode of lymphopenia over the study period, compared with $6.5 \%$ of controls (online supplemental table 3). Infection risk was increased in individuals with lymphopenia without IBD (HR 1.26 (95\% CI 1.17 to 1.36$), \mathrm{p}<0.001)$ but not in those with IBD (HR 1.05 (95\% CI 0.97 to 1.14), $\mathrm{p}=0.21$ ) (online supplemental table $3 \mathrm{~A})$. Results were consistent when $\mathrm{UC}$ and $\mathrm{CD}$ were evaluated in separate models (online supplemental table 3B,C). Online supplemental table $4 \mathrm{~A}$ summarises the absolute numbers of people with and without IBD and recorded infection events according to lymphocyte count categories ( severe $\left(<0.5 \times 10^{9}\right.$ cells $\left./ \mathrm{L}\right)$, moderate $(0.5-0.8)$, mild $(0.8-1.0)$, normal (1.0-4.0), lymphocytosis $(\geq 4.0)$ ).

Figure 3 also shows that, in continuous analysis, a higher neutrophil count was associated with an increased risk of common infection both in the IBD and the non-IBD cohorts. However, there was no evidence of an increased risk of infection in association with lower neutrophil counts. When categorised by neutrophil count status (neutrophil count $<1.5 \times 10^{9}$ cells $/ \mathrm{L}$ vs $\geq 1.5 \times 10^{9}$ cells $/ \mathrm{L}$ ), $1.9 \%$ of people with IBD and $1.5 \%$ of those without IBD had at least one count in the neutropenic range over follow-up. There was no evidence of an association with infection for either group (HR for IBD 1.12 (95\% CI 0.80 to 1.55 ), $\mathrm{p}=0.51$, HR for controls 1.05 (95\% CI 0.87 to 1.27), $\mathrm{p}=0.60$ ) (online supplemental table $3 \mathrm{~A}$ ). When UC and $\mathrm{CD}$ were evaluated in separate models, there was no evidence of an association between neutropenia status and risk of infection (UC HR 1.17 (95\% CI 0.78 to 1.77), $\mathrm{p}=0.45)$, (CD HR 1.06 (95\% CI 0.61 to 1.93$), \mathrm{p}=0.84$ ) (online supplemental table 3B,C). Online supplemental table 4B summarises the absolute numbers in those with and without IBD and recorded infection events according to the neutrophil count categories (severe $\left(<0.5 \times 10^{9}\right.$ cells $\left./ \mathrm{L}\right)$, moderate $(0.5-1.0)$, mild $(1.0-1.5)$, normal (1.5-7.5), neutrophilia (>7.5)). Continuous associations for lymphocyte and neutrophil counts were near 
Table 1 Covariate summary statistics for ulcerative colitis, Crohn's disease and control cohorts

\begin{tabular}{|c|c|c|c|c|}
\hline & $\begin{array}{l}\text { With CD } \\
(\mathrm{n}=7469)\end{array}$ & $\begin{array}{l}\text { Matched cohort } \\
\text { without CD }(n=29 \\
876)\end{array}$ & $\begin{array}{l}\text { With UC }(n=11 \\
360)\end{array}$ & $\begin{array}{l}\text { Matched } \\
\text { cohort } \\
\text { without UC } \\
(n=45440)\end{array}$ \\
\hline Age at study entry (years) Median (IQR) & $47(34,61)$ & $48(33,63)$ & $53(39,67)$ & $52(37,67)$ \\
\hline Male sex & $3402(45.5)$ & $14085(47.1)$ & $5737(50.5)$ & $22433(49.4$ \\
\hline $\begin{array}{l}\text { Time since GP practice registration (years) median } \\
\text { (IQR) }\end{array}$ & $10(2,20)$ & $11(3,20)$ & $11(3,21)$ & $11(3,21)$ \\
\hline Duration of IBD (years) median (IQR) & $9(2,17)$ & NA & $9(2,18)$ & NA \\
\hline \multicolumn{5}{|l|}{ Ethnicity } \\
\hline Asian & $247(4.2)$ & $1402(6.0)$ & $547(6.1)$ & $2239(6.3)$ \\
\hline Black & $72(1.2)$ & $599(2.6)$ & $93(1.0)$ & $811(2.3)$ \\
\hline Mixed & $55(0.9)$ & $246(1.1)$ & $63(0.7)$ & $361(1.0)$ \\
\hline Other & $41(0.7)$ & $235(1.0)$ & $68(0.8)$ & $299(0.8)$ \\
\hline White & $5399(92.9)$ & 20732 (89.3) & $8270(91.5)$ & $32058(89.6$ \\
\hline \multicolumn{5}{|l|}{ BMI category $\left(\mathrm{kg} / \mathrm{m}^{2}\right)$} \\
\hline Underweight $(\leq 18.5)$ & $369(4.9)$ & $800(2.7)$ & $256(2.3)$ & $1094(2.4)$ \\
\hline Normal weight (18.5-25) & $3020(40.4)$ & $10170(34.0)$ & $4230(37.2)$ & $14905(32.8$ \\
\hline Overweight (25-30) & $2125(28.5)$ & $8819(29.5)$ & 3684 (32.4) & $14399(31.7$ \\
\hline Obese $(\geq 30)$ & $1304(17.5)$ & $6307(21.1)$ & $2223(19.6)$ & $9744(21.4)$ \\
\hline BMI not recorded & $651(8.7)$ & $3780(12.7)$ & 967 (8.5) & $5298(11.7)$ \\
\hline \multicolumn{5}{|l|}{ Smoking status } \\
\hline Non-smoker & $2641(35.4)$ & 12511 (41.9) & $4255(37.5)$ & $18288(40.2$ \\
\hline Current smoker & $1731(23.2)$ & $6135(20.5)$ & $1386(12.2)$ & 8881 (19.5) \\
\hline Ex-smoker & $3028(40.5)$ & $10632(35.6)$ & $5631(49.6)$ & $17594(38.7)$ \\
\hline Smoking status not recorded & $69(0.9)$ & $598(2.0)$ & $88(0.8)$ & $677(1.5)$ \\
\hline \multicolumn{5}{|l|}{ Index of multiple deprivation quintile } \\
\hline 1 (most deprived) & 1015 (13.6) & $4130(13.8)$ & $1293(11.4)$ & $5534(12.2)$ \\
\hline 2 & $1185(15.9)$ & 4738 (15.9) & $1626(14.3)$ & $6694(14.7)$ \\
\hline 3 & 1484 (19.9) & 5867 (19.6) & $2143(18.9)$ & 8779 (19.3) \\
\hline 4 & $1765(23.6)$ & $6840(22.9)$ & $2862(25.2)$ & $11024(24.3$ \\
\hline 5 (least deprived) & $1860(24.9)$ & 7704 (25.8) & $3230(28.4)$ & $12579(27.7)$ \\
\hline IMD not recorded & $160(2.1)$ & $597(2.0)$ & $206(1.8)$ & $830(1.8)$ \\
\hline \multicolumn{5}{|l|}{ Alcohol intake } \\
\hline Non-drinker & $1158(15.5)$ & $3945(13.2)$ & 1495 (13.2) & $5799(12.8)$ \\
\hline Within limits & 4178 (55.9) & $16449(55.1)$ & $6724(59.2)$ & $25880(57.0$ \\
\hline Over recommended limits & $991(13.3)$ & $4390(14.7)$ & 1691 (14.9) & $6882(15.1)$ \\
\hline Alcoholism & $119(1.6)$ & $505(1.7)$ & $172(1.5)$ & $774(1.7)$ \\
\hline Alcohol intake not recorded & $1023(13.7)$ & $4587(15.4)$ & $1278(11.2)$ & $6105(13.4)$ \\
\hline \multicolumn{5}{|l|}{ Comorbidities } \\
\hline Hypertension & $1292(17.3)$ & $5894(19.7)$ & $2704(23.8)$ & $10929(24.1)$ \\
\hline Hyperlipidaemia & $1391(18.6)$ & $7462(25.0)$ & $3293(29.0)$ & $13480(29.7)$ \\
\hline Type two diabetes & $436(5.8)$ & $1992(6.7)$ & $1052(9.3)$ & $3674(8.1)$ \\
\hline Peripheral vascular disease & $81(1.1)$ & $276(0.9)$ & $139(1.2)$ & $584(1.3)$ \\
\hline Atrial fibrillation & $172(2.3)$ & $797(2.7)$ & $388(3.4)$ & 1479 (3.3) \\
\hline Myocardial infarction & $172(2.3)$ & $694(2.3)$ & $424(3.7)$ & 1360 (3.0) \\
\hline Angina & $139(1.9)$ & $531(1.8)$ & $337(3.0)$ & $1101(2.4)$ \\
\hline
\end{tabular}




\begin{tabular}{|c|c|c|c|c|}
\hline & $\begin{array}{l}\text { With CD } \\
(n=7469)\end{array}$ & $\begin{array}{l}\text { Matched cohort } \\
\text { without } C D(n=29 \\
876)\end{array}$ & $\begin{array}{l}\text { With UC (n=11 } \\
360)\end{array}$ & $\begin{array}{l}\text { Matched } \\
\text { cohort } \\
\text { without UC } \\
\text { ( } n=45440 \text { ) }\end{array}$ \\
\hline Stroke & $119(1.6)$ & $430(1.4)$ & $226(2.0)$ & $831(1.8)$ \\
\hline Heart failure & $87(1.2)$ & $322(1.1)$ & $179(1.6)$ & $675(1.5)$ \\
\hline CKD stages $3-5$ & $406(5.4)$ & $1344(4.5)$ & $796(7.0)$ & $2897(6.4)$ \\
\hline COPD & $111(1.5)$ & $179(0.6)$ & $235(2.1)$ & $285(0.6)$ \\
\hline Chronic liver disease & $364(4.9)$ & $922(3.1)$ & 489 (4.3) & $1696(3.7)$ \\
\hline Malignancy & $315(4.2)$ & $1308(4.4)$ & $689(6.1)$ & $2479(5.5)$ \\
\hline Dementia & $136(1.8)$ & $611(2.0)$ & $292(2.6)$ & $1230(2.7)$ \\
\hline Rheumatoid arthritis & $85(1.1)$ & $194(0.6)$ & $157(1.4)$ & $383(0.8)$ \\
\hline History of fracture & $612(8.2)$ & 2371 (7.9) & $1017(9.0)$ & $3721(8.2)$ \\
\hline Depression & $1160(15.5)$ & $3091(10.3)$ & $1461(12.9)$ & $4786(10.5)$ \\
\hline \multicolumn{5}{|l|}{ Baseline medication use } \\
\hline Rectal 5-ASA & $329(4.4)$ & $25(0.1)$ & $2415(21.3)$ & $35(0.1)$ \\
\hline Rectal glucocorticoids & $340(4.6)$ & $263(0.9)$ & $1793(15.8)$ & $395(0.9)$ \\
\hline Oral 5-ASA & $2868(38.4)$ & $97(0.3)$ & $6986(61.5)$ & $212(0.5)$ \\
\hline Oral glucocorticoids & $2290(30.7)$ & $1780(6.0)$ & 2937 (25.9) & $3103(6.8)$ \\
\hline Non-biologic immunosuppressants & $2274(30.4)$ & $207(0.7)$ & $1600(14.1)$ & $377(0.8)$ \\
\hline Biologic therapies & $182(2.4)$ & $6(0.0)$ & $59(0.5)$ & $7(0.0)$ \\
\hline
\end{tabular}

Data are $\mathrm{N}(\%)$ unless stated.

5-ASA, aminosalicylic acid medications; BMI, body mass index; CD, Crohn's disease; CKD, chronic kidney disease; COPD, chronic obstructive pulmonary disease; GP, general practitioner; IBD, inflammatory bowel disease; UC, ulcerative colitis.

identical when counts in the 2 months prior to an infection were excluded (online supplemental figure 1).

\section{Sensitivity analysis}

Results for the primary outcomes of common infection, viral infection and GI infection were consistent in all sensitivity analyses for both UC and CD (1) excluding controls who were practice nonattendees prior to study start, (2) excluding people with IBD registered within 1 year of study start, (3) without adjustment for medication use in multivariable models, applying a comorbidity count score instead of adjustment for individual comorbidities; including only patients with complete data on sociodemographic and behavioural characteristics (online supplemental table 5).

\section{DISCUSSION}

This study showed that people with IBD are more likely to present to primary care with common infections including URTI, acute bronchitis, skin infection, GI infection, herpes zoster, UTI (UC only) and pneumonia (CD only) than matched controls. Treatment with oral glucocorticoid therapy, immunotherapies and biologic therapy, but not with 5-ASA, was associated with additional infection risk. Although mild lymphopenia and neutropenia were more common in individuals with IBD than controls, these were not generally associated with significantly increased infection risk in IBD.

To the best of our knowledge, this is the first study evaluating the risk of a range of infections in people with IBD performed in primary care. Previous studies have largely focused on opportunistic infections or drug-resistant organisms in secondary care settings. ${ }^{36-38}$ Similar to this study, Long et al found a greater risk for pneumonia in CD (HR 1.71, 95\% CI 1.62 to 1.80 ) than for UC (HR 1.41, $95 \%$ CI 1.34 to 1.48$).{ }^{13}$ The increased risk for herpes zoster in this study was similar to that found by Gupta $e t$ al (UC incidence rate ratio: 1.21 (95\% CI, 1.05 to 1.40 ), CD incidence rate ratio: 1.61 (95\% CI, 1.35 to 1.92$)$ ) ${ }^{14}$ suggesting the greatest excess risk is in CD. Ning et al demonstrated similar associations in their meta-analysis. ${ }^{39}$ In this study, we describe an increase in GI infections in both $\mathrm{UC}$ and $\mathrm{CD}$, with the majority being clinically diagnosed. However, a subanalysis of stool culture-confirmed diagnoses of infection with Salmonella, Shigella, Campylobacter or Clostridium difficile confirmed that this finding was not confounded by the fact that active IBD could be mistaken for GI infection. Previous studies have similarly shown GI infections are more common in people with IBD. ${ }^{40-45}$ Rodemann et al observed a threefold increased rate of Clostridium difficile infection among people with IBD in secondary care ${ }^{41}$ and Singh et al reported a 4.8fold increased risk for Clostridium difficile infection in IBD 
Table 2 Associations between ulcerative colitis and Crohn's disease, and each infection outcome.

\begin{tabular}{|c|c|c|c|c|c|}
\hline \multirow{2}{*}{$\begin{array}{l}\text { Number of } \\
\text { patients }\end{array}$} & \multirow{2}{*}{$\begin{array}{l}\text { Patient } \\
\text { years at- } \\
\text { risk }\end{array}$} & \multirow[b]{2}{*}{ Events (\%) } & \multirow{2}{*}{$\begin{array}{l}\text { Event rate } \\
\text { (per 100-person } \\
\text { years) }\end{array}$} & \multicolumn{2}{|c|}{ Hazard ratio $(95 \% \mathrm{Cl})$} \\
\hline & & & & Unadjusted & Adjusted $^{*}$ \\
\hline
\end{tabular}

A. Associations between ulcerative colitis and each infection outcome

\begin{tabular}{|c|c|c|c|c|c|c|}
\hline \multicolumn{7}{|c|}{ Any common infection } \\
\hline Controls & 45440 & 133703 & $17403(38 \%)$ & $13.0(12.8,13.2)$ & 1.00 (ref) & 1.00 (ref) \\
\hline Ulcerative colitis & 11360 & 31076 & $5111(45 \%)$ & $16.4(16.0,16.9)$ & $1.25(1.21 \text { to } 1.29)^{*}$ & $1.12(1.07$ to 1.18$)$ \\
\hline \multicolumn{7}{|c|}{ Gastrointestinal infection } \\
\hline Controls & 45440 & 134615 & $1365(3 \%)$ & $1.0(0.9,1.1)$ & 1.00 (ref) & 1.00 (ref) \\
\hline Ulcerative colitis & 11360 & 31410 & $510(4 \%)$ & $1.6(1.5,1.8)$ & $1.61(1.45 \text { to } 1.78)^{*}$ & $1.46(1.26$ to 1.69$)$ \\
\hline \multicolumn{7}{|l|}{ Viral infection } \\
\hline Controls & 45440 & 136290 & $8510(19 \%)$ & $6.2(6.1,6.4)$ & 1.00 (ref) & 1.00 (ref) \\
\hline Ulcerative colitis & 11360 & 31994 & $2544(22 \%)$ & $8.0(7.6,8.3)$ & $1.27(1.21 \text { to } 1.33)^{*}$ & $1.13(1.05$ to 1.20$)$ \\
\hline
\end{tabular}

B. Associations between Crohn's disease and each infection outcome

Any common infection

\begin{tabular}{|c|c|c|c|c|c|c|}
\hline Controls & 29876 & 88753 & $11227(38 \%)$ & $12.7(12.4,12.9)$ & 1.00 (ref) & 1.00 (ref) \\
\hline Crohn's disease & 7469 & 19892 & $3470(46 \%)$ & $17.4(16.9,18.0)$ & $1.36(1.31 \text { to } 1.41)^{*}$ & $1.20(1.14 \text { to } 1.26)^{*}$ \\
\hline \multicolumn{7}{|c|}{ Gastrointestinal infection } \\
\hline Crohn's disease & 7469 & 20170 & $395(5 \%)$ & $2.0(1.8,2.2)$ & $2.09(1.86 \text { to } 2.36)^{*}$ & $1.83(1.56 \text { to } 2.15)^{*}$ \\
\hline \multicolumn{7}{|l|}{ Viral infection } \\
\hline
\end{tabular}

${ }^{*} \mathrm{p}<0.001$.

*Models adjusted for age, sex, IMD quintile, white ethnicity, BMI category, smoking status, alcohol category, hypertension, hyperlipidaemia, type 2 diabetes, peripheral arterial disease, atrial fibrillation, angina, myocardial infarction, stroke, heart failure, chronic kidney disease stage 3-5, chronic obstructive pulmonary disorder, chronic liver disease, malignancy, dementia, rheumatoid arthritis, fracture history, depression and concomitant medication use (from rectal 5-ASA, rectal glucocorticoids, oral 5-ASA, oral glucocorticoids, immunotherapies and biologic therapies).

ASA, aminosalicylic acid; BMI, body mass index; IMD, index of multiple deprivation.

in primary care. ${ }^{42}$ The increased risk of GI infection may relate to interconnected local factors such as gut dysbiosis, impaired gut epithelial barrier repair, dysfunctional immune regulation and chronic intestinal inflammation. These factors also play key roles in IBD pathogenesis, particularly in genetically susceptible individuals. $546-48$

We also found that a wide range of infections was more common in UC and CD than in controls, even after adjustment for sociodemographic factors, comorbidities and baseline medication use. Medications that suppress the immune system are the most widely recognised risk factor for infection in IBD. In addition, an increased prevalence in IBD of other risk factors for infection including malnutrition, surgery and increased pathogen exposure $^{3649}$ could contribute to the observed increase in infection rates. Pathogenic processes, such as penetrating disease, which occurs in a proportion of individuals with $\mathrm{CD}$, may further contribute to this risk. Finally, low vaccine uptake and, perhaps, an impaired response to vaccines may compound infection risk in IBD. ${ }^{50}$

Oral glucocorticoid therapy, immunotherapies and biologic therapy were associated with additional infection risk in this study. Use of glucocorticoids in people with IBD was common $(35.3 \%)$, as found previously in primary care studies of other immune-mediated inflammatory diseases such as rheumatoid arthritis. ${ }^{51} 52$ While prospective data from the IBD cancer and serious infections in Europe (I-CARE) study are awaited ${ }^{53}$ several studies have examined the impact of medications on infection risk in both UC and CD. ${ }^{711} 125455$ Toruner et al found in a secondary care setting that the risk of opportunistic infection in IBD increased with the use of glucocorticoids (OR $2.2,95 \%$ CI 1.0 to 4.9 ), azathioprine/6-mercaptopurine (OR 3.4, 95\% CI 1.5 to 7.5) and infliximab (OR 11.1, 95\% CI 0.8 to 148 ); the greatest risk was observed when these medications were used in combination. ${ }^{7}$ A similar risk for opportunistic infection associated with antitumour necrosis factor- $\alpha$ (TNF $\alpha)$ therapy was shown in a metaanalysis by Ford et al. ${ }^{54}$ Similarly, Long et al showed that corticosteroids (OR 1.73, 95\% CI 1.51 to 1.99 ), anti-TNFo (OR $1.81,95 \%$ CI 1.48 to 2.21 ) and thiopurines (OR $1.85,95 \%$ CI 1.61 to 2.13 ) were independent risk factors for herpes zoster in IBD, with the greatest association being for combined thiopurine and anti-TNF $\alpha$ therapy 


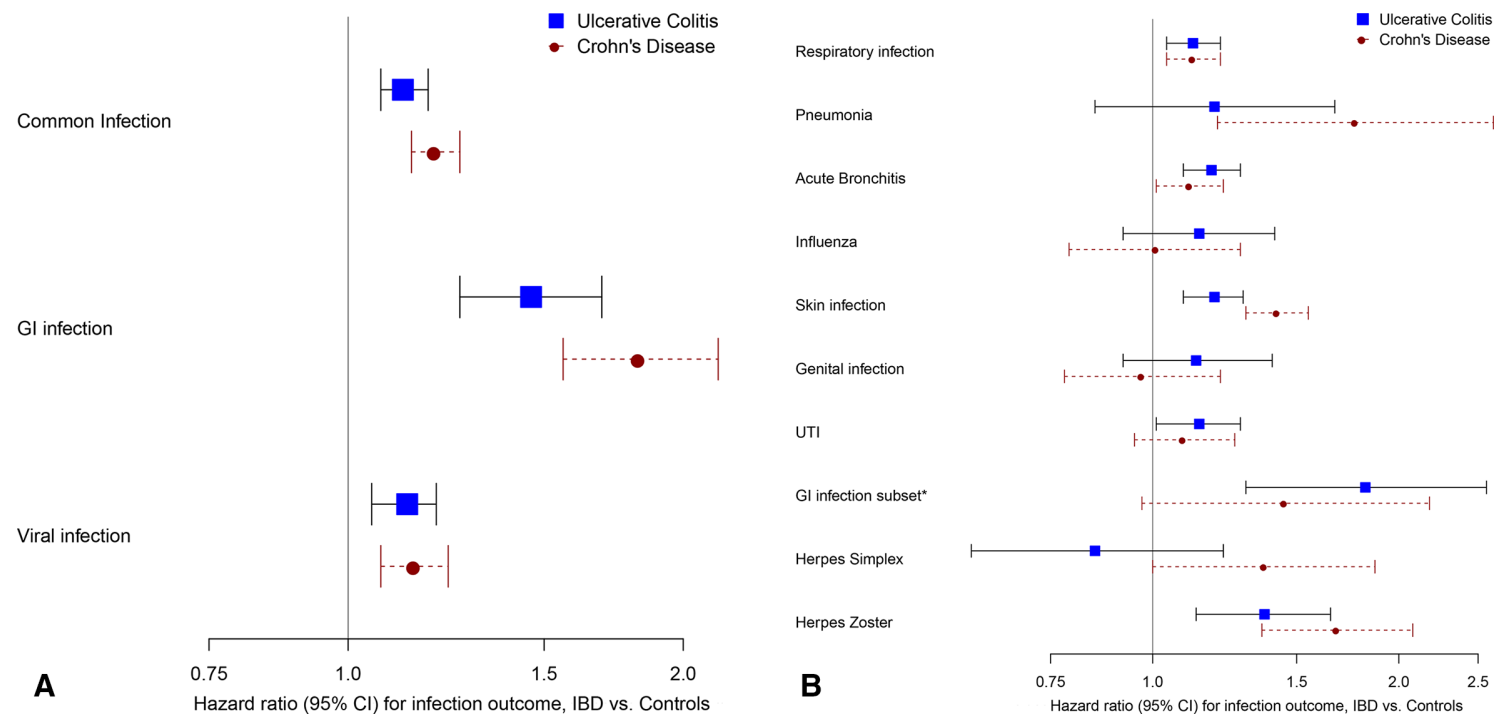

(OR 3.29, 95\% CI 2.33 to 4.65) ${ }^{56}$ Increased herpes zoster incidence has also been observed in people taking tofacitinib, a Janus kinase inhibitor recently licensed for use in moderate to severe UC. ${ }^{57}{ }^{58}$ Conversely, oral and rectal 5-ASA medications do not appear to increase infection risk in this study. This is also consistent with the findings of Toruner et al who found no association between 5-ASA use and opportunistic infections, ${ }^{7}$ adding to the data confirming the long-term safety profile of 5-ASA. The association between immunosuppressive drugs and biologics with infection needs to be interpreted in terms of the progressive and destructive nature of uncontrolled inflammation in IBD, which can result in complications such as fibrosis, stenosis and malignancy. Furthermore, active IBD is associated with anxiety and depression and may have socioeconomic repercussions that affect quality of life. Patient concordance with effective therapy is, therefore, paramount, and an association between these drugs and an increased risk of infection should not prevent their use where appropriate.

We did not show an association between mild lymphopenia or neutropenia and increased infection risk in IBD, an interesting finding that warrants further study. The finding that lower lymphocyte counts were associated with a decreased chance of infection in CD might correlate with thiopurine use and consequent lower disease activity. Similarly, a retrospective observational study from Germany found no association between mild lymphopenia and opportunistic infections, ${ }^{59}$ while Toruner et al found thiopurine-induced lymphopenia or neutropenia in IBD did not increase opportunistic infections. ${ }^{7}$ These findings suggest that either IBD itself or the drugs used to treat it result in, or are associated with, immune dysfunction and/or dysregulation that influences infection risk independent of either neutropenia or lymphopenia. Recent studies have indicated that innate lymphoid cells play a key role in mucosal inflammation and immunity against bacterial, viral and parasitic infection, and that an imbalanced accumulation in the population of Th1, Th2, Th17 and natural killer cells not only perpetuates chronic inflammation but also causes a dysfunctional immune response to infection in IBD. ${ }^{48}$ It is also likely that additional inhibition of innate and adaptive immunity by drugs used to treat IBD could exacerbate immune and/or cytokine imbalance, contributing to the observed infection risk.

A key strength of this study is the use of the highest quality of real-world data on infectious diseases from the RCGP RSC network, the UK primary care infections surveillance network. The infections explored are part of 34 regularly monitored conditions within the network. Results were consistent in all sensitivity analysis, including exclusion of matched controls who were potential practice nonattendees (a likely over-adjustment). Nonlinear continuous risk models were used in our primary analysis to explore the association between time-updated lymphocyte and neutrophil count and risk of infection, avoiding the need for subgroups defined by the presence or absence of a haematological abnormality. This continuous modelling approach maximises power by avoiding arbitrary dichotomisation and enables our results to be generalisable, given the variability in normal reference range values for 
Rectal 5-ASA

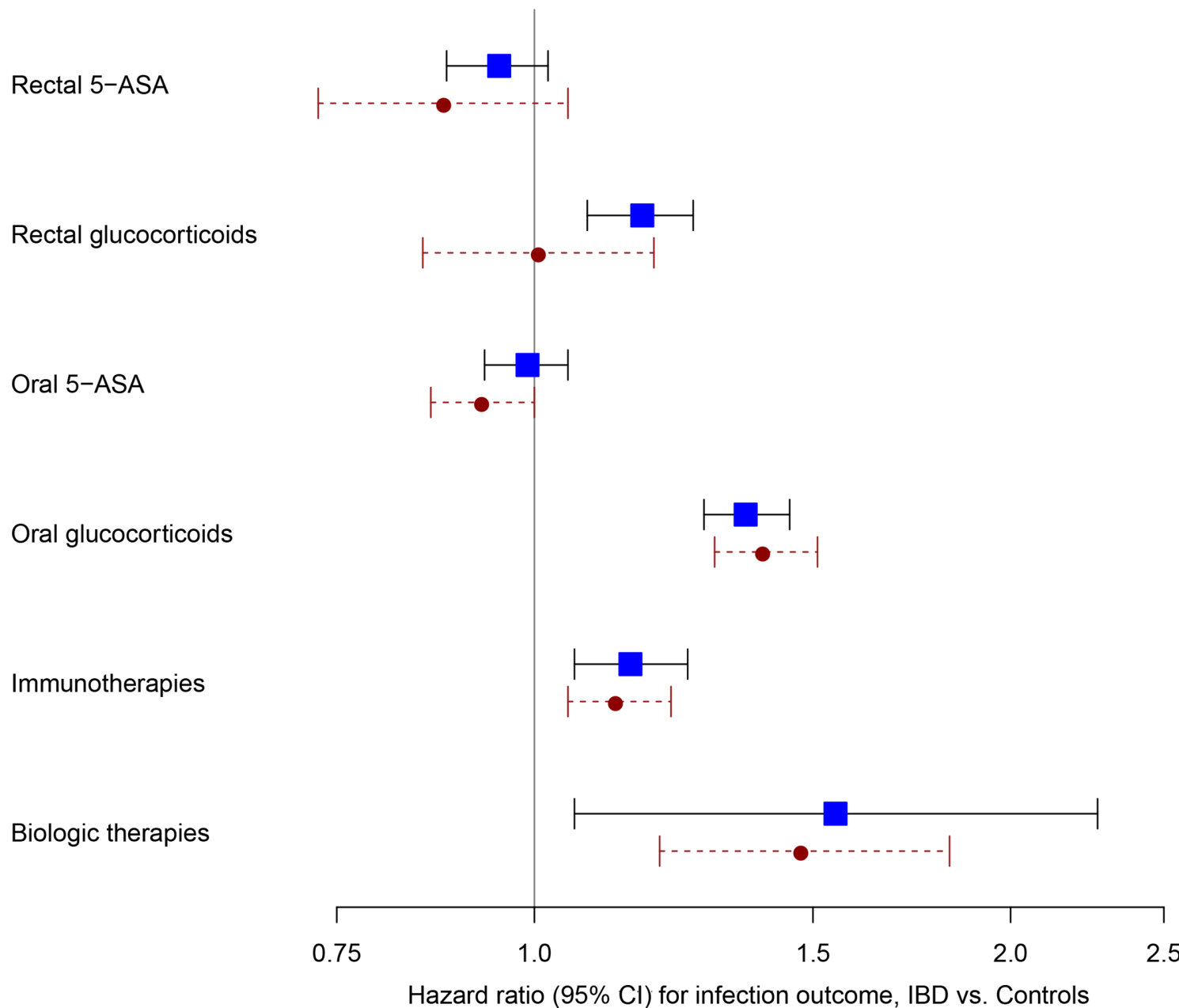

Rectal glucocorticoids

Ulcerative Colitis

- Crohn's Disease

Figure 2 Adjusted hazard ratios for associations between baseline medication use and subsequent risk of presentation of common infection in individuals with ulcerative colitis and Crohn's disease. A HR greater than 1 represents an increase in the risk of presentation of infection in individuals using the medication of interest compared with individuals not using that medication. Models adjusted for age, sex, IMD quintile, white ethnicity, BMI category, smoking status, alcohol category, hypertension, hyperlipidaemia, type 2 diabetes, peripheral arterial disease, atrial fibrillation, angina, myocardial infarction, stroke, heart failure, chronic kidney disease stage 3-5, chronic obstructive pulmonary disorder, chronic liver disease, malignancy, dementia, rheumatoid arthritis, fracture history, depression and concomitant medication use (from rectal 5-ASA, rectal glucocorticoids, oral 5-ASA, oral glucocorticoids, immunotherapies and biologic therapies). ASA, aminosalicylic acid; BMI, body mass index; GI,gastrointestinal; IBD, inflammatory bowel disease; IMD, index of multiple deprivation.

haematological parameters in different settings. ${ }^{60}$ As with all observational studies of this type, a limitation of our analysis is that we were unable to determine any causal associations, and although results were consistent with extensive sensitivity analysis we cannot rule out unobserved confounding as an explanation of our findings, especially given the relatively modest effect sizes observed. Data recording and capture bias might be another study limitation, as symptomatic individuals with IBD may have specific patterns of care meaning they present more frequently to clinicians, who may also investigate infections in people with IBD more proactively. A further potential bias is delayed IBD diagnosis meaning the approach of retaining controls who later becomes a case in the control set meant some person-time in the controls comprised people with IBD, although we expect the effect of this to be a minor attenuation of effect sizes towards the null. Although infections diagnosed in secondary care are often transferred into primary care records, this study is likely to have undercaptured such diagnoses that were made or treated solely in secondary care. In addition, biological therapies in the UK are normally prescribed in secondary care and may not be recorded by primary care, which will have resulted in systemic undercapture of biologic use. This issue stems from the lack of linkage between the different data systems used in UK primary and secondary care. UK electronic health record data do not capture the underlying reason for the prescription, meaning we cannot ascertain the 

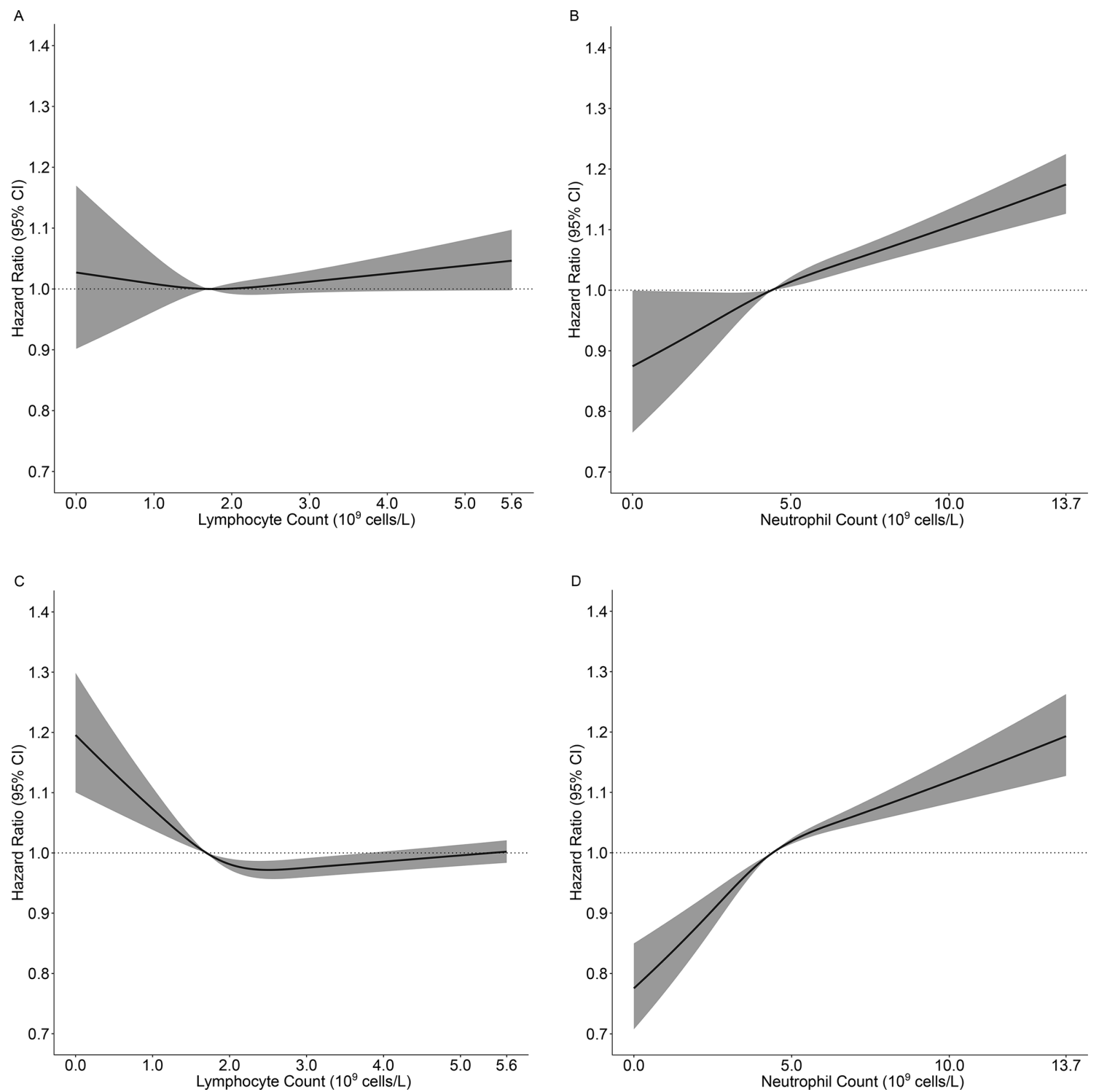

Figure 3 Association of continuous time-varying lymphocyte and neutrophil count with risk of presentation of common infection in individuals with and without inflammatory bowel disease. Lymphocyte and neutrophil counts each modelled a restricted cubic spline with three knots in a multivariable model ${ }^{*}$. Associations are shown relative to the mean count in individuals with IBD (lymphocyte count $1.7 \times 10^{9} \mathrm{cell} / \mathrm{s} / \mathrm{L}$; neutrophil count $4.4 \times 10^{9} \mathrm{cell} / \mathrm{s} / \mathrm{L}$ ), up to the 99.5 th centile of each count variable. Grey shading represents $95 \%$ Cls. *Models adjusted for age, sex, IMD quintile, white ethnicity, BMI category, smoking status, alcohol category, hypertension, hyperlipidaemia, type 2 diabetes, peripheral arterial disease, atrial fibrillation, angina, myocardial infarction, stroke, heart failure, chronic kidney disease stage 3-5, chronic obstructive pulmonary disorder, chronic liver disease, malignancy, dementia, rheumatoid arthritis, fracture history, depression and medication use (rectal 5-ASA, rectal glucocorticoids, oral 5-ASA, oral glucocorticoids, immunotherapies and biologic therapies. With IBD $(n=15290$, infection events=6692), (A) lymphocyte count and (B) neutrophil count. Controls ( $n=44324$, infection events=16 332), (C) lymphocyte count and (D) neutrophil count. ASA, aminosalicylic acid; BMI, body mass index; IBD, inflammatory bowel disease; IMD, index of multiple deprivation.

true indication of the medications evaluated in the study. While we explored associations between baseline medication exposure and risk of infection, these only represent a snapshot of a patient's medication exposure. For this reason, our estimates of medication use are lower than other studies, in particular, a recent analysis by Chhaya et al that assesses cumulative medication use in the 5 years after an IBD diagnosis. ${ }^{61}$ We did not assess dosage effects, unlike a previous study that showed a higher risk of common infection in people prescribed higher initial doses of glucocorticoid treatment. ${ }^{62}$ A time-updated evaluation of the effect of cumulative medication exposure and medication dose on infection risk in IBD, as well as potential drug-by-drug interactions, would be of interest for future work. Finally, this study did not show associations with all the infection subtypes examined. ILI and genital infections were not more common in individuals with IBD. However, the paucity of literature exploring these subtypes in IBD prevents further comparisons. 


\section{CONCLUSIONS}

People with IBD are at a higher risk of presenting with a wide range of diagnosed infections compared with matched controls of the same age and gender without IBD. The findings of this study suggest clinicians must be vigilant for infections in IBD, particularly in the context of use of systemic corticosteroids, immunotherapies and biologic agents. As highlighted by the recent COVID-19 pandemic, this is of importance in people who have comorbidities. Infection risk mitigation should be a multifactorial, pre-emptive approach involving early preventative screening (including appropriate vaccination), ${ }^{36} 43$ and optimisation of nutritional status ${ }^{63}$ and comorbidities. Further research is necessary to elucidate the mechanisms underlying specific infection susceptibility in the IBD population, the role of infection in the IBD pathological process, and the effect(s) of long-term use of immunomodulatory therapeutics on the course of IBD and on outcomes.

Acknowledgements Patients and practices who are members of the 0xfordRoyal College of General Practitioners (RCGP) Research and Surveillance Centre (RSC) network, who allow their data to be shared for surveillance, research, quality improvement and education. The collaboration of primary care computerised medical record system providers EMIS, TPP, InPractice Systems and Wellbeing in facilitating the RCGP RSC data. Medical writing and statistical support, funded by Pfizer, were provided by John Dennis, Andrew McGovern, and Annick Moon (Momentum Data), with project management support from Filipa Ferreira (University of Oxford)

Contributors KB, SdeL, DT, MN and PMI designed the study, supervised the data analysis, provided the interpretation of results and contributed to the drafting and critical review of the manuscript. All authors approved the final draft. As corresponding author, PMI attests that all listed authors meet authorship criteria and that no others meeting the criteria have been omitted. PMI is the guarantor and accepts full responsibility for the conduct of the study, had access to the data and controlled the decision to publish.

Funding This study, and medical writing support, was funded by Pfizer. As study authors, representatives of Pfizer contributed to the design and conduct of the study, interpretation of the data; preparation, review, or approval of the manuscript; and decision to submit the manuscript for publication.

Competing interests KB has received honoraria from Tillots, Thermo Fisher Scientific, Boehringer Ingelheim, Pfizer, and Yakult. SdeL is director of the RCGP RSC, he has received funding through his universities from Eli Lilly, GSK, Astra Zeneca, MSD, Sanofi, Seqirus, and Takeda. DT and MN are employees of Pfizer. PMI has received lecture fees from AbbVie, Celgene, Falk Pharma, Ferring, MSD, Janssen, Pfizer, Takeda, Tillotts, Sapphire Medical, Sandoz, Shire, and Warner Chilcott; financial support for research from MSD, Pfizer, and Takeda; advisory fees from AbbVie, Arena, Genentech, Gilead, Hospira, Janssen, Lilly, MSD, Pfizer, Pharmacosmos, Prometheus, Roche, Sandoz, Samsung Bioepis, Takeda, Topivert, VH2, Vifor Pharma, and Warner Chilcott.

Patient consent for publication Not required.

Ethics approval Study approval was granted by the Research Committee of the RCGP RSC. The study did not require a formal ethics board review, as defined using the NHS Health Research Authority research decision tool (http://www.hradecisiontools.org.uk/research/).

Provenance and peer review Not commissioned; externally peer reviewed.

Data availability statement Data may be obtained from a third party and are not publicly available. The RCGP RSC dataset is held securely at Oxford University and the University of Surrey and can be accessed by bonefide researchers. Approval is on a project-by-project basis (www.rcgp.org.uk/rsc). Ethical approval by an NHS Research Ethics Committee may be needed before any data release/other appropriate approval. Researchers wishing to directly analyse the patient-level pseudonymised data will be required to complete information governance training and work on the data from university secure servers. Patient-level data cannot be taken out of the secure network.
Supplemental material This content has been supplied by the author(s). It has not been vetted by BMJ Publishing Group Limited (BMJ) and may not have been peer-reviewed. Any opinions or recommendations discussed are solely those of the author(s) and are not endorsed by BMJ. BMJ disclaims all liability and responsibility arising from any reliance placed on the content. Where the content includes any translated material, BMJ does not warrant the accuracy and reliability of the translations (including but not limited to local regulations, clinical guidelines, terminology, drug names and drug dosages), and is not responsible for any error and/or omissions arising from translation and adaptation or otherwise.

Open access This is an open access article distributed in accordance with the Creative Commons Attribution Non Commercial (CC BY-NC 4.0) license, which permits others to distribute, remix, adapt, build upon this work non-commercially, and license their derivative works on different terms, provided the original work is properly cited, appropriate credit is given, any changes made indicated, and the use is non-commercial. See: http://creativecommons.org/licenses/by-nc/4.0/.

ORCID iD

Peter M Irving http://orcid.org/0000-0003-0972-8148

\section{REFERENCES}

1 Baumgart DC, Sandborn WJ. Inflammatory bowel disease: clinical aspects and established and evolving therapies. Lancet 2007;369:1641-57

2 Bodger K. Cost of illness of Crohn's disease. Pharmacoeconomics 2002;20:639-52.

3 Bassi A, Dodd S, Williamson P, et al. Cost of illness of inflammatory bowel disease in the UK: a single centre retrospective study. Gut 2004;53:1471-8.

$4 \mathrm{Ng} \mathrm{SC}$, Shi HY, Hamidi N, et al. Worldwide incidence and prevalence of inflammatory bowel disease in the 21 st century: a systematic review of population-based studies. Lancet 2018;390:2769-78.

5 Neurath MF. Targeting immune cell circuits and trafficking in inflammatory bowel disease. Nat Immunol 2019;20:970-9.

6 Kucharzik T, Maaser C. Infections and chronic inflammatory bowel disease. Viszeralmedizin 2014;30:6-32.

7 Toruner M, Loftus EV, Harmsen WS, et al. Risk factors for opportunistic infections in patients with inflammatory bowel disease. Gastroenterology 2008;134:929-36.

8 Kennedy NA, Jones G-R, Lamb CA, et al. British Society of gastroenterology guidance for management of inflammatory bowel disease during the COVID-19 pandemic. Gut 2020;69:984-90.

9 Crooks CJ, West J, Card TR. A comparison of the recording of comorbidity in primary and secondary care by using the Charlson index to predict short-term and long-term survival in a routine linked data cohort. BMJ Open 2015;5:e007974.

10 Brassard P, Bitton A, Suissa A, et al. Oral corticosteroids and the risk of serious infections in patients with elderlyonset inflammatory bowel diseases. Am J Gastroenterol 2014;109:109:1795-802.

11 Lichtenstein GR, Feagan BG, Cohen RD, et al. Serious infections and mortality in association with therapies for Crohn's disease: treat registry. Clin Gastroenterol Hepatol 2006;4:621-30.

12 Lichtenstein GR, Feagan BG, Cohen RD, et al. Serious infection and mortality in patients with Crohn's disease: more than 5 years of follow-up in the TREAT'M registry. Am $J$ Gastroenterol 2012;107:1409-22.

13 Long MD, Martin C, Sandler RS, et al. Increased risk of pneumonia among patients with inflammatory bowel disease. Am J Gastroenterol 2013;108:240-8.

14 Gupta G, Lautenbach E, Lewis JD. Incidence and risk factors for herpes zoster among patients with inflammatory bowel disease. Clin Gastroenterol Hepatol 2006;4:1483-90.

15 Warny M, Helby J, Nordestgaard BG, et al. Lymphopenia and risk of infection and infection-related death in 98,344 individuals from a prospective Danish population-based study. PLoS Med 2018;15:e1002685.

16 Irving P, Barrett K, Tang D, et al. Common infections, mental health problems and healthcare use in people with inflammatory bowel disease: a cohort study protocol. Evid Based Ment Health 2020. doi:10.1136/ebmental-2020-300167. [Epub ahead of print: 17 Sep 2020].

17 Correa A, Hinton W, McGovern A, et al. Royal College of general practitioners research and surveillance centre (RCGP RSC) sentinel network: a cohort profile. BMJ Open 2016;6:e011092.

18 de Lusignan S, Liaw S-T, Michalakidis G, et al. Defining datasets and creating data dictionaries for quality improvement and research in 
chronic disease using routinely collected data: an ontology-driven approach. Inform Prim Care 2011;19:127-34.

19 de Lusignan S, van Weel C. The use of routinely collected computer data for research in primary care: opportunities and challenges. Fam Pract 2006;23:253-63.

20 de Lusignan S, Metsemakers JF, Houwink P, et al. Routinely collected general practice data: goldmines for research? A report of the European Federation for medical informatics primary care informatics Working Group (EFMI PCIWG) from MIE2006, Maastricht, the Netherlands. Inform Prim Care 2006;14:203-9.

21 Nikiphorou E, de Lusignan S, Mallen CD, et al. Cardiovascular risk factors and outcomes in early rheumatoid arthritis: a populationbased study. Heart 2020;106:1566-72.

22 Williams R, Alexander G, Armstrong I, et al. Disease burden and costs from excess alcohol consumption, obesity, and viral hepatitis: fourth report of the Lancet standing Commission on liver disease in the UK. Lancet 2018;391:1097-107.

23 Woodmansey C, McGovern AP, McCullough KA, et al. Incidence, demographics, and clinical characteristics of diabetes of the exocrine pancreas (type 3C): a retrospective cohort study. Diabetes Care 2017;40:1486-93.

24 Galloway J, Barrett K, Irving P, et al. Risk of venous thromboembolism in immune-mediated inflammatory diseases: a UK matched cohort study. RMD Open 2020;6:e001392.

25 de Lusignan S, Correa A, Pathirannehelage S, et al. RCGP research and surveillance centre annual report 2014-2015: disparities in presentations to primary care. Br J Gen Pract 2017;67:e29-40.

26 Amirthalingam G, Andrews N, Keel P, et al. Evaluation of the effect of the herpes zoster vaccination programme 3 years after its introduction in England: a population-based study. Lancet Public Health 2018;3:e82-90.

27 Pathirannehelage S, Kumarapeli P, Byford R, et al. Uptake of a Dashboard designed to give RealTime feedback to a sentinel network about key data required for influenza vaccine effectiveness studies. Stud Health Technol Inform 2018;247:161-5.

28 Ananthakrishnan AN, Cai T, Savova G, et al. Improving case definition of Crohn's disease and ulcerative colitis in electronic medical records using natural language processing: a novel informatics approach. Inflamm Bowel Dis 2013;19:1411-20.

29 Stapley SA, Rubin GP, Alsina D, et al. Clinical features of bowel disease in patients aged $<50$ years in primary care: a large casecontrol study. Br J Gen Pract 2017;67:e336-44.

30 Abrahami D, Douros A, Yin H, et al. Dipeptidyl peptidase-4 inhibitors and incidence of inflammatory bowel disease among patients with type 2 diabetes: population based cohort study. BMJ 2018;360:k872.

31 Department for Communities and Local Government. The English indices of deprivation. 2015, 2015.

32 Tippu Z, Correa A, Liyanage $\mathrm{H}$, et al. Ethnicity recording in primary care computerised medical record systems: an ontological approach. J Innov Health Inform 2017;23:920:799.

33 Newburger PE, Dale DC. Evaluation and management of patients with isolated neutropenia. Semin Hematol 2013;50:198-206.

34 Denaxas SC, George J, Herrett E, et al. Data resource profile: cardiovascular disease research using linked bespoke studies and electronic health records (caliber). Int J Epidemiol 2012;41:1625-38.

35 Therneau TM, Grambsch PM. Modeling survival data: extending the Cox model. Berlin: Springer Science \& Business Media, 2000.

36 Rahier JF, Magro F, Abreu C, et al. Second European evidencebased consensus on the prevention, diagnosis and management of opportunistic infections in inflammatory bowel disease. J Crohns Colitis 2014;8:443-68.

37 Nguyen GC, Leung W, Weizman AV. Increased risk of vancomycinresistant Enterococcus (VRE) infection among patients hospitalized for inflammatory bowel disease in the United States. Inflamm Bowel Dis 2011;17:1338-42.

38 Nguyen GC, Patel H, Chong RY. Increased prevalence of and associated mortality with methicillin-resistant Staphylococcus aureus among hospitalized IBD patients. Am J Gastroenterol 2010;105:371-7.

39 Ning L, Liu R, Li S, et al. Increased risk of herpes zoster infection in patients with inflammatory bowel disease: a meta-analysis of cohort studies. Eur J Clin Microbiol Infect Dis 2020;39:219-27.
40 Limsrivilai J, Saleh ZM, Johnson LA, et al. Prevalence and effect of intestinal infections detected by a PCR-based stool test in patients with inflammatory bowel disease. Dig Dis Sci 2020;65:3287-96.

41 Rodemann JF, Dubberke ER, Reske KA, et al. Incidence of Clostridium difficile infection in inflammatory bowel disease. Clin Gastroenterol Hepatol 2007;5:339-44.

42 Singh H, Nugent Z, Yu BN, et al. Higher incidence of Clostridium difficile infection among individuals with inflammatory bowel disease. Gastroenterology 2017;153:430-8.

43 Lamb CA, Kennedy NA, Raine T, et al. British Society of gastroenterology consensus guidelines on the management of inflammatory bowel disease in adults. Gut 2019;68:s1-106.

44 Axelrad JE, Joelson A, Green PHR, et al. Enteric infections are common in patients with flares of inflammatory bowel disease. Am J Gastroenterol 2018;113:1530-9.

45 Hanada Y, Khanna S, Loftus EV, et al. Non-Clostridium difficile bacterial infections are rare in patients with flares of inflammatory bowel disease. Clin Gastroenterol Hepatol 2018;16:528-33.

46 Rahman FZ, Marks DJB, Hayee BH, et al. Phagocyte dysfunction and inflammatory bowel disease. Inflamm Bowel Dis 2008;14:1443-52.

47 Xu X-R, Liu C-Q, Feng B-S, et al. Dysregulation of mucosal immune response in pathogenesis of inflammatory bowel disease. World $\mathrm{J}$ Gastroenterol 2014;20:3255-64.

48 Wallace KL, Zheng L-B, Kanazawa Y, et al. Immunopathology of inflammatory bowel disease. World J Gastroenterol 2014;20:6-21.

49 Ananthakrishnan AN, McGinley EL. Infection-related hospitalizations are associated with increased mortality in patients with inflammatory bowel diseases. J Crohns Colitis 2013;7:107-12.

50 Irving PM, Gibson PR. Infections and IBD. Nat Clin Pract Gastroenterol Hepatol 2008;5:18-27.

51 Nikiphorou E, de Lusignan S, Mallen CD, et al. Cardiovascular risk factors and outcomes in early rheumatoid arthritis: a populationbased study. Heart 2020;106:heartjnl-2019-316193.

52 Nikiphorou E, de Lusignan S, Mallen C. Haematological abnormalities in new-onset rheumatoid arthritis and risk of common infections: a population-based study. Rheumatology2019.

53 The I-Care Study Group. P509 IBD cancer and serious infections in Europe (I-care): a European prospective observational study. Journal of Crohn's and Colitis 2017;11:S338-9.

54 Ford AC, Peyrin-Biroulet L. Opportunistic infections with antitumor necrosis factor- $\alpha$ therapy in inflammatory bowel disease: meta-analysis of randomized controlled trials. Am J Gastroenterol 2013;108:1268-76.

55 Kirchgesner J, Lemaitre M, Carrat F, et al. Risk of serious and opportunistic infections associated with treatment of inflammatory bowel diseases. Gastroenterology 2018;155:337-46. e10.

56 Long MD, Martin C, Sandler RS, et al. Increased risk of herpes zoster among 108604 patients with inflammatory bowel disease. Aliment Pharmacol Ther 2013;37:420-9.

57 Colombel J-F. Herpes zoster in patients receiving JAK inhibitors for ulcerative colitis: mechanism, epidemiology, management, and prevention. Inflamm Bowel Dis 2018;24:2173-82.

58 Winthrop KL, Melmed GY, Vermeire S, et al. Herpes zoster infection in patients with ulcerative colitis receiving tofacitinib. Inflamm Bowel Dis 2018;24:2258-65.

59 Vögelin M, Biedermann L, Frei P, et al. The impact of AzathioprineAssociated lymphopenia on the onset of opportunistic infections in patients with inflammatory bowel disease. PLoS One 2016;11:e0155218

60 Coates S, Wang D, Pierscionek T, et al. Time- and race-specific haematological reference intervals for healthy volunteer trials: a retrospective analysis of pooled data from multiple phase I trials. Front Pharmacol 2020;11:314.

61 Chhaya V, Saxena S, Cecil E, et al. Steroid dependency and trends in prescribing for inflammatory bowel disease - a 20-year national population-based study. Aliment Pharmacol Ther 2016;44:482-94.

62 Fardet L, Petersen I, Nazareth I. Common infections in patients prescribed systemic glucocorticoids in primary care: a populationbased cohort study. PLoS Med 2016;13:e1002024.

63 Forbes A, Escher J, Hébuterne X, et al. ESPEN guideline: clinical nutrition in inflammatory bowel disease. Clin Nutr 2017;36:321-47. 